\title{
Enhanced Degradation of Lactide-co-Glycolide Polymer with Basic Nucleophilic Drugs
}

\author{
Susan D’Souza, ${ }^{1}$ Jabar A. Faraj, ${ }^{2}$ Rossella Dorati, ${ }^{3}$ and Patrick P. DeLuca ${ }^{4}$ \\ ${ }^{1}$ Sunovion Pharmaceuticals Inc., Marlborough, MA 01752, USA \\ ${ }^{2}$ Evonik Inc., 750 Lakeshore Parkway, Birmingham, AL 35211, USA \\ ${ }^{3}$ Section of Pharmaceutical Chemistry, Department of Drug Sciences, University of Pavia, Viale Taramelli 12, 27100 Pavia, Italy \\ ${ }^{4}$ University of Kentucky College of Pharmacy, Lexington, KY 40536, USA
}

Correspondence should be addressed to Susan D’Souza; dr_ssdsouza@yahoo.com

Received 10 August 2014; Accepted 4 December 2014

Academic Editor: Mohammad F. Kiani

Copyright (C) 2015 Susan D’Souza et al. This is an open access article distributed under the Creative Commons Attribution License, which permits unrestricted use, distribution, and reproduction in any medium, provided the original work is properly cited.

\begin{abstract}
The purpose of this study was to examine the degradative effect of weakly basic nucleophilic drugs on a lactide-co-glycolide (PLGA) polymer in a microsphere formulation. Biodegradable PLGA microspheres of two second-generation atypical antipsychotics, Risperidone and Olanzapine, were manufactured using a solvent extraction/evaporation technique. The effect of drug content, buffer $\mathrm{pH}$ and temperature on polymer molecular weight and degradation, were examined via a series of experiments and compared against a control (Placebo PLGA microspheres). In comparison to Placebo microspheres, significant polymer molecular weight reduction was observed upon encapsulation of varying levels of either Risperidone or Olanzapine. There was excellent correlation between the extent of molecular weight reduction during manufacture and the amount of encapsulated drug in the microspheres. Subsequent studies on polymer degradation showed: the following (a) the Placebo and Olanzapine microspheres followed pseudo first order kinetics, (b) Risperidone microspheres exhibited biphasic degradation profiles, and (c) polymer degradation was dependent on temperature, not $\mathrm{pH}$. The findings of these studies show that encapsulation of weakly basic nucleophile type drugs into PLGA can accelerate the biodegradation of the PLGA and have major implications on the design of polymeric microsphere drug delivery systems.
\end{abstract}

\section{Introduction}

Considerable advances in the areas of research involving biodegradable polymers as implantable reservoirs for sustainedrelease drug delivery have been realized over the past few decades [1-4]. A key feature that makes use of biodegradable polymers attractive in the medical sciences is the fact that they do not require surgical removal after completion of drug release. Among the most popular biodegradable polymers are polylactide (PLA) and poly(lactic-co-glycolide) (PLGA) polymers, which are known to be nontoxic, biocompatible, and nonimmunogenic.

Generally, degradation of poly(esters) like PLA and PLGA occurs in four major stages:

(a) polymer hydration causing disruption of the primary and secondary structure due to hydrogen bonding and van der Waals forces, (b) loss of mechanical strength caused by the rupture of covalent bonds of the polymer backbone to form oligomers with acidic end-groups,

(c) polymer mass loss due to diffusion of acidic oligomers resulting in accelerated water absorption,

(d) polymer dissolution and/or phagocytosis [5].

As would be expected, polymer hydration of PLA or PLGA causes ester hydrolysis that has been shown to proceed via pseudo first order kinetics and is described as follows:

$$
\frac{\mathrm{d}[\mathrm{COOH}]}{\mathrm{dt}}=k[\mathrm{COOH}],
$$

where $[\mathrm{COOH}]$ refers to the concentration of the carboxylic acid end group in the PLA or PLGA polymer and " $k$ " is the rate constant. Eventually, hydrolytic degradation of 


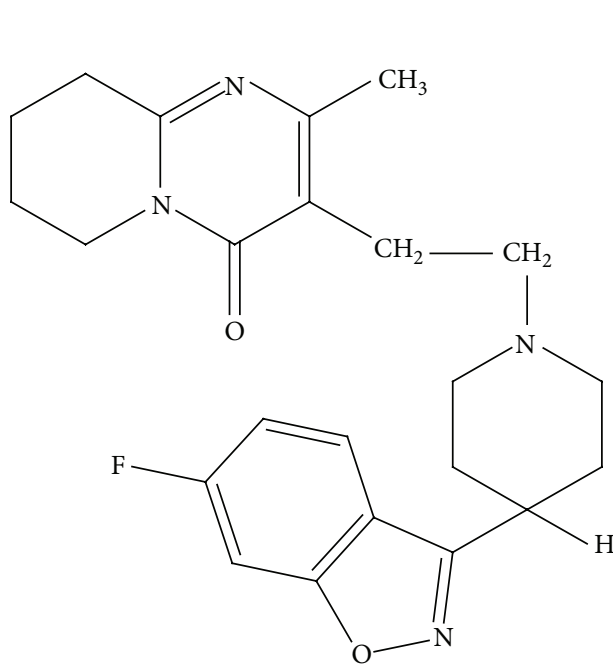

Risperidone

(a)

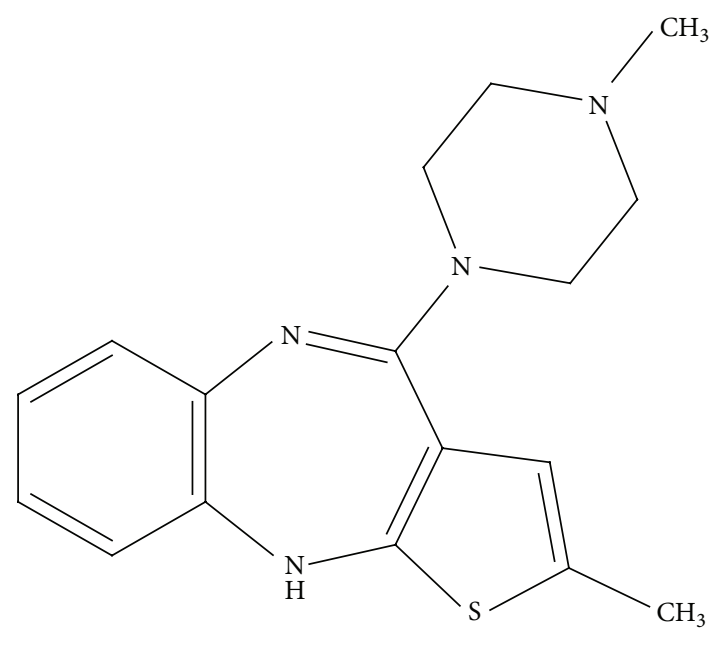

Olanzapine

(b)

FIgURE 1: Chemical structures of Risperidone and Olanzapine.

the poly(ester) produces lactic and glycolic acids, both moieties cleared in vivo by the Krebs cycle.

In addition to their use in surgical sutures and prosthetic devices, biodegradable poly(ester) polymers are also used in commercially available injectable products, for example, Risperdal Consta (Risperidone in PLGA) and Lupron Depot (Leuprolide acetate in PLGA) [6, 7]. Literature also cites examples of a number of therapeutic agents like small molecules, peptides, and proteins that have been successfully encapsulated into these polymers $[8,9]$. Depending on the polymer type and the incorporated therapeutic agent, the polymeric dosage form can provide sustained drug levels for varying durations in vivo $[10,11]$. Indeed, research has shown that these therapeutic agents have varying physicochemical properties that could alter polymer degradation and thereby alter drug release profiles [12-16]. This highlights the need to investigate the effect of a drug on polymer degradation as it can be critical with respect to product performance, as cited by several authors [17-19].

While the chemical composition of PLGA and PLA is essential for biodegradability and biocompatibility, a notable effect on the drug release profile is detected especially if the therapeutic agent is a basic drug. Such types of drugs are expected to interact with the acidic polymer thereby accelerating polymer degradation and influencing drug release from the polymer matrix. For instance, researchers have reported that tertiary amine drugs or nucleophilic compounds like local anesthetics accelerate the hydrolytic degradation of poly(D,L-lactide) polymer [20-22]. These aspects illustrate that studies involving design and development of a biodegradable microsphere dosage form must include studies on possible degradative effects of the drug on the polymer. Such studies will not only aid in the fundamental understanding of the dosage form but also enable advancements in the area of polymeric delivery systems.
Towards this goal, the research presented in this publication explores the degradation in microsphere formulations containing basic drugs. The drugs chosen for the study are two popular second-generation atypical antipsychotics, Risperidone and Olanzapine. Both molecules are commonly prescribed for the treatment of schizophrenia and other psychotic disorders [23-26]. The structures of Risperidone and Olanzapine are shown in Figure 1. Both drugs are weak bases having pKa values of 8.24 and 7.37 for Risperidone and Olanzapine, respectively. Risperidone contains two tertiary amines while Olanzapine contains two tertiary amines and one secondary amine suggesting that the latter drug is a stronger base and nucleophile. The work reported in this publication details the approach taken during the development of microsphere formulations of Risperidone and Olanzapine and showcases the effects of drug loading, temperature, and $\mathrm{pH}$ on the degradation of a $65: 35$ PLGA polymer.

The objectives of this study include:

(i) identification of any possible effects of Risperidone and Olanzapine on the PLGA polymer,

(ii) assessment of the influence of drug loading on polymer degradation,

(iii) determination of the impact of buffer and temperature on the polymer degradation process.

The study also highlights the similarities and differences in behavior between Olanzapine and Risperidone and provides a framework for researchers investigating sustained dosage formulations that utilize biodegradable polymers.

\section{Materials and Methods}

2.1. Materials. Hydrochloride salts of Risperidone and Olanzapine were purchased from Cipla Ltd., Bombay, India, DL65: 35 PLGA $\left(\mathrm{MW}_{\text {initial }}=75 \mathrm{kDa}\right)$ from Medisorb, Dupont, 
Wilmington, DE, and Polyvinyl alcohol (PVA, MW 30$70 \mathrm{kDa}$ ) from Sigma, St. Louis, MO, USA. All other chemicals were obtained commercially as analytical grade reagents.

2.2. Preparation of Microspheres. Placebo, Risperidone, and Olanzapine PLGA microspheres were prepared by dispersing a homogenous solution of polymer and drug into an aqueous solution containing $0.35 \%$ Polyvinyl alcohol followed by solvent extraction/evaporation for 2 hours [27, 28]. The solidified microspheres were recovered by filtration and dried under vacuum at room temperature for 48 hours.

2.3. Drug Content. A $10 \mathrm{mg}$ amount of microspheres was dissolved in $8 \mathrm{~mL}$ Acetonitrile followed by the addition of $32 \mathrm{~mL}$ of $0.1 \mathrm{M}$ acetate buffer, $\mathrm{pH}$ 4.0. The contents were diluted with $80: 20$ Acetate buffer: acetonitrile mixture and filtered using $0.45 \mu \mathrm{m}$ Teflon filter, prior to analysis. The two drugs were assayed by reverse phase HPLC method using a Nucleosil $5 \mu \mathrm{C}_{18}$ column $(250 \times 4.6 \mathrm{~mm}$; Phenomenex, Torrance, CA, USA), mobile phase: $70: 30$ water : acetonitrile with $0.1 \%$ TFA, flow rate $=1 \mathrm{~mL} / \mathrm{min}$ at $275 \mathrm{~nm}$.

2.4. Particle Size Distribution. Particles were sized by laser diffractometry using a Malvern 2600 laser sizer (Malvern 2600 particle sizer, Malvern, UK). The average particle size was expressed as the volume mean diameter $\left(V_{\mathrm{md}}\right)$ in microns.

2.5. Degradation of Microspheres. $50 \mathrm{mg}$ of drug loaded microspheres or Placebo microspheres was transferred to a bottle containing $50 \mathrm{~mL}$ of $0.02 \mathrm{M}$ phosphate buffer saline (PBS) $\mathrm{pH}$ 7.2 containing $0.05 \%$ Tween 80 , at $37^{\circ} \mathrm{C}$. At predetermined intervals, the remaining microspheres were filtered using $0.65 \mu \mathrm{m}$ PVDF filters (Millipore, USA). The microspheres were dried in a vacuum drier for at least 3 days to ensure low moisture content followed by determination of molecular weight.

2.6. Determination of Molecular Weight. The molecular weight distribution of Placebo and drug loaded microspheres was determined by gel permeation chromatography (GPC). The GPC system consisted of two Ultrastyragel columns connected in series $\left(7.8 \times 300 \mathrm{~mm}\right.$ each, one with $10^{4} \AA$ pores and one with $10^{3} \AA$ pores), a delivery device (Shimadzu LC-6A, Japan), UV detector set at $\lambda=210 \mathrm{~nm}$ (Shimadzu, Japan), and software to compute molecular weight distribution (Waters, Maxima 820, Milford, USA). Sample solutions in tetrahydrofuran (THF) at a concentration of $5 \mathrm{mg} / \mathrm{mL}$ were filtered through a $0.45 \mu \mathrm{m}$ filter (Millipore, USA) before injection into the GPC system and were eluted with THF at $0.4 \mathrm{~mL} / \mathrm{min}$. The weight-average molecular weight was calculated using monodisperse polystyrene standard where

(i) $\mathrm{MW}_{\text {initial }}$ is the initial polymer molecular weight $(75 \mathrm{kDa})$,

(ii) $\mathrm{MW}_{0}$ represents the molecular weight after manufacture of Placebo or drug loaded microspheres ( $t$-zero),

(iii) $\mathrm{MW}_{t}$ at time " $t=x$ days" is the molecular weight of a sample (Placebo or drug loaded microspheres).
TABLE 1: Mean particle size of Risperidone and Olanzapine microspheres.

\begin{tabular}{lcc}
\hline Drug loading (\%) & $\begin{array}{c}\text { Risperidone } \\
\text { microspheres } \\
(\mu \mathrm{m})\end{array}$ & $\begin{array}{c}\text { Olanzapine } \\
\text { microspheres } \\
(\mu \mathrm{m})\end{array}$ \\
\hline 1 & 8.80 & 10.4 \\
5 & 12.7 & 9.80 \\
10 & 15.4 & 12.3 \\
15 & 23.4 & 10.0 \\
35 & 20.8 & - \\
\hline
\end{tabular}

\section{Results and Discussion}

3.1. Drug Content and Particle Size Distribution. Drug content for all the batches of Risperidone and Olanzapine microspheres was assessed by HPLC and the results are described in Table 1. Both drugs were easily encapsulated into the PLGA polymer at a range of 1-15\% loading for Olanzapine and $1-35 \%$ for Risperidone. Thus, at lower loadings, the microsphere formulations had a low drug to polymer ratio, while at higher loadings the drug to polymer ratio was high. Intuitively, this would suggest a greater influence of drug on any drug-polymer interaction at higher loadings.

Results of particle size analysis for Risperidone and Olanzapine microspheres, as measured by laser diffractometry, are listed in Table 1. Mean particle size for Olanzapine microspheres remained unchanged, irrespective of drug loading level. In contrast, there is a threefold increase in mean particle size at higher loadings of Risperidone $(8.8-23.4 \mu \mathrm{m})$. Since the particle size of Risperidone and Olanzapine microspheres at different loadings was small $(<25 \mu \mathrm{m})$, particle size was not expected to significantly contribute to polymer degradation, especially at particle sizes $<15 \mu \mathrm{m}$.

3.2. Polymer Degradation in Placebo and Drug Loaded Microspheres. In vitro polymer degradation studies were carried out on the Placebo, Risperidone, and Olanzapine microspheres in $0.02 \mathrm{M}$ PBS, pH 7.2 containing $0.05 \%$ Tween 80 , at $37^{\circ} \mathrm{C}$, over 15 days. Using GPC, polymer molecular weight of samples was analyzed at $t$-zero $\left(\mathrm{MW}_{0}\right)$, as well as predetermined time-points, for example, $t=2,5,10$, and 15 days $\left(\mathrm{MW}_{t}\right)$, for all the microspheres.

3.2.1. Effect of Risperidone on Polymer Degradation. The effect of 1-35\% Risperidone loading on degradation of the $65: 35$ PLGA polymer in $0.02 \mathrm{M}$ phospahte buffer saline (PBS) $\mathrm{pH}$ 7.2 containing $0.05 \%$ Tween 80 , at $37^{\circ} \mathrm{C}$, is presented as a semilogarithmic plot in Figure 2. Unlike the Placebo microspheres where molecular weight was unchanged at $t$-zero $\left(\mathrm{MW}_{\text {initial }}=\right.$ $\mathrm{MW}_{0}$ ), with the Risperidone loaded microspheres, a substantial reduction in molecular weight was observed within 2 days $\left(\mathrm{MW}_{2}\right)$, after which degradation rates slowed considerably. Thus, the degradation profile of the Risperidone microspheres was biphasic. The rapid initial decrease (2 days) in polymer molecular weight was ascribed to an attack from the weakly basic drug to the acidic polymer, followed by a plateau possibly due to neutralization of the weakly basic drug 


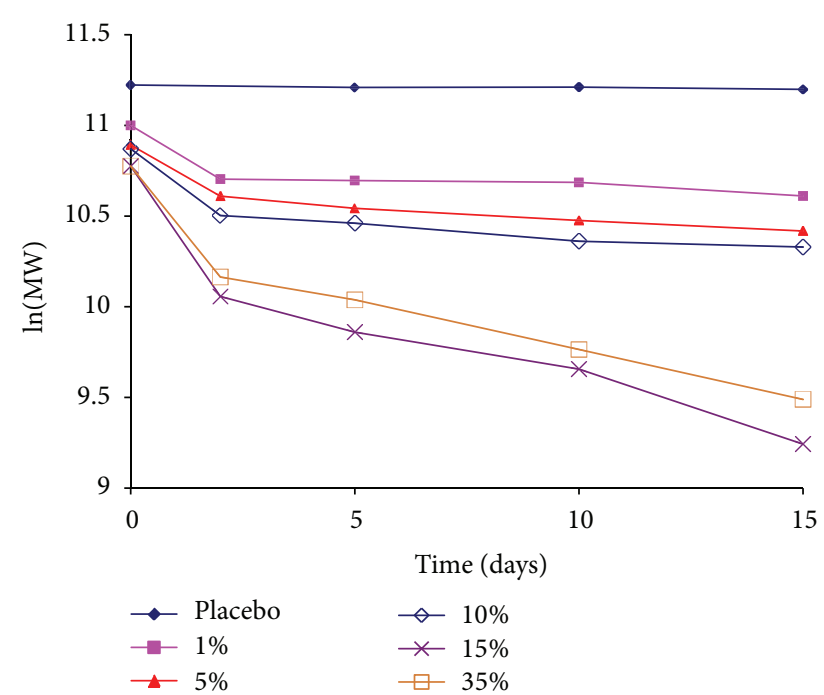

FIGURE 2: Effect of Risperidone loading on polymer degradation.

with acids formed during polymer hydration. Thus, after 2 days, the degradation rate for the $1 \%$ loaded microspheres was similar to the Placebo microspheres, while the 5\% and $10 \%$ loading showed only slightly faster degradation rates. At higher loadings of Risperidone, ratio of Risperidone to polymer was high; the loss in molecular weight was ascribed to catalytic effects of the drug on polymer degradation, even though some degree of neutralization of the basic drug was expected. For the 15 and 35\% loaded microspheres, the molecular weight reduction after 2 days was the highest $(\sim 50 \%)$ and the degradation continued to about $80-85 \%$ reduction after 15 days. As such, the degradation profiles with the 15 and 35\% were quite close, with $15 \%$ loading causing slightly greater degradation. These findings suggest that the catalytic effects caused by the tertiary amine groups in Risperidone reach a plateau at high loadings (15-35\%).

Results from this study indicate that Risperidone loading had a profound effect on polymer molecular weight reduction at two distinct stages:

(a) after manufacture of the microspheres $\left(\mathrm{MW}_{0}<\right.$ $\left.\mathrm{MW}_{\text {initial }}\right)$,

(b) within 2 days of incubation in PBS $\left(\mathrm{MW}_{2}<\mathrm{MW}_{0}\right)$, followed by less pronounced effects after day 2 of the in vitro degradation study.

3.2.2. Effect of Olanzapine on Polymer Degradation. The impact of Olanzapine on degradation of the 65:35 PLGA polymer in $0.02 \mathrm{M}$ phosphate buffer saline (PBS) pH 7.2 containing $0.05 \%$ Tween 80 , at $37^{\circ} \mathrm{C}$, is shown as a semilogarithmic plot in Figure 3. Rapid polymer degradation was observed at all the loadings (1-15\%). From Figure 3, it is evident that the degradation rate with Olanzapine followed pseudo first order kinetics, similar to that of the Placebo, albeit much faster. In contrast with Risperidone, where some drug neutralization effects were observed after day 2 with $1 \%$ loaded microspheres (lowest loading), the presence of a reactive secondary amine in Olanzapine ensured continual

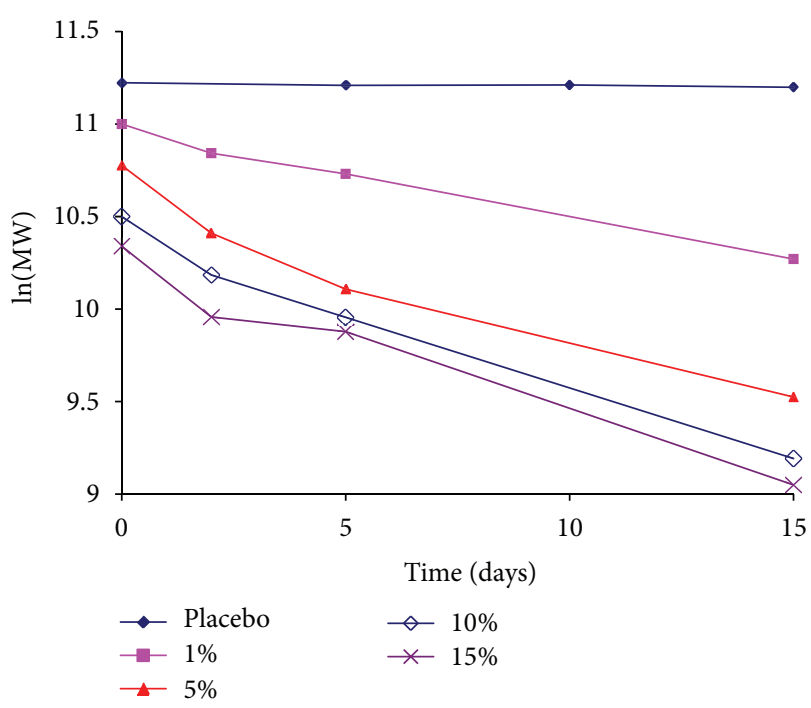

FIGURE 3: Effect of Olanzapine loading on polymer degradation.

degradation of the PLGA polymer even at the lowest drug load. Notably, the degradation rate for all Olanzapine loaded microspheres was high, and greater than that observed with Risperidone as well as Placebo microspheres. Thus, it is reasonable to assert that, due to the nature and type of chemical functionalities in a molecule such as Olanzapine, it would be difficult to control the degradation of a poly(ester) type polymer like PLGA, even when low amounts of drug levels are incorporated in the microspheres. Overall, the polymer degradation profile observed with Olanzapine microspheres was much faster than that observed for Risperidone. Indeed, at loadings between 5 and 15\% Olanzapine, the molecular weight reduction at 15 days was approximately $81-88 \%$ of $\mathrm{MW}_{\text {initial }}$.

Results from this study confirm that, for Olanzapine, substantial molecular weight reduction was noted at two distinct phases:

(a) after manufacture of the microspheres $\left(\mathrm{MW}_{0}<\right.$ $\mathrm{MW}_{\text {initial }}$ ), similar to Risperidone,

(b) throughout the in vitro degradation study.

3.2.3. Kinetics of Polymer Degradation. As mentioned in the introduction (refer to Section 1), PLGA hydrolysis follows pseudo first order kinetics. However, data from Figures 2 and 3 reveal a biphasic degradation profile for the Risperidone microspheres in $0.02 \mathrm{M}$ phosphate buffer saline (PBS) $\mathrm{pH}$ 7.2 containing $0.05 \%$ Tween 80 , at $37^{\circ} \mathrm{C}$, but not the Olanzapine and Placebo microspheres. The biphasic degradation profile indicates two varying polymer degradation rates after incorporation of Risperidone into the biodegradable PLGA microsphere (Figure 2). Indeed, a marked difference was observed between the degradation in Region 1 ( 0 to 2 days) and Region 2 (after 2 days till day 15), where Region 1 depicts a much faster degradation rate in comparison to Region 2. This contrasting behavior is ascribed to the effect of Risperidone on the PLGA polymer. Hence, biphasic polymer degradation constants $\left(k_{1}, k_{2}\right)$ and half-life $\left(t_{1 / 2(1)}, t_{1 / 2(2)}\right)$ 
TABle 2: Rate constant and half-life for Placebo, Olanzapine, and Risperidone microspheres.

\begin{tabular}{|c|c|c|c|c|c|c|c|c|c|c|}
\hline \multirow{3}{*}{ Parameter measured } & \multicolumn{10}{|c|}{$\%$ drug loading } \\
\hline & \multirow{2}{*}{$\begin{array}{c}0 \\
\text { Placebo }\end{array}$} & \multicolumn{2}{|c|}{1} & \multicolumn{2}{|c|}{5} & \multicolumn{2}{|c|}{10} & \multicolumn{2}{|c|}{15} & \multirow{2}{*}{$\begin{array}{c}35 \\
\text { Risp. }\end{array}$} \\
\hline & & Risp. & Olanz. & Risp. & Olanz. & Risp. & Olanz. & Risp. & Olanz. & \\
\hline$k$ or $k_{1}\left(\right.$ day $\left.^{-1}\right)$ & 0.003 & 0.148 & 0.047 & 0.141 & 0.077 & 0.184 & 0.083 & 0.359 & 0.080 & 0.306 \\
\hline$t_{1 / 2}$ or $t_{1 / 2(1)}$ (day) & 213 & 4.68 & 14.6 & 4.91 & 8.97 & 3.77 & 8.35 & 1.93 & 8.63 & 2.267 \\
\hline$k_{2}\left(\right.$ day $\left.^{-1}\right)$ & - & 0.007 & - & 0.014 & - & 0.014 & - & 0.060 & - & 0.053 \\
\hline$t_{1 / 2(2)}$ (day) & - & 102.3 & - & 48.1 & - & 49.5 & - & 11.5 & - & 13.2 \\
\hline
\end{tabular}

values for Region 1 and Region 2 were calculated and are shown in Table 2.

In Region 1, rate constants for Risperidone appeared relatively constant (between 0.141 and 0.148 /day) through $5 \%$ loading, after which higher loadings led to higher $k_{1}$ values, with a plateau in degradation rate at the $15-35 \%$ loading range (Table 2). Higher $k_{1}$ values at higher loadings are not surprising as there is a direct relationship between the increased degradation rate and reduced polymer to drug ratio in the Risperidone microspheres. In contrast, $k_{2}$ values for Risperidone were significantly lower than $k_{1}$ values, with a low value of $0.007 /$ day at $1 \%$ loading, doubling to 0.014 /day for the 5 and $10 \%$ loadings to reach a plateau around 0.05 $0.06 /$ day at loadings greater than $15 \%$ Risperidone $\left(R^{2}>0.95\right.$ obtained for $k_{2}$ at all drug loadings). Given that the sampling time points for Regions 1 and 2 were predetermined, calculations of rate constants in Region 1 (Table 2) are reasonable estimates. It should be noted that even if additional data were collected in Region 1, the overall profile of polymer degradation used to measure the rate constant $k_{1}$ would not be markedly different than what was observed in Figure 2.

Using the $k$ values in Table 2, the half-life values for Regions 1 and 2 for both Risperidone loaded microspheres were calculated from the corresponding rate constants. From Table 2, it is evident that half-life values in Region 1 are faster than those in Region 2, in some cases by a few orders of magnitude. For Risperidone, $t_{1 / 2(1)}$ values (1-15\% loadings) in Region 1 ranged from 1.93 to 4.91 days and increased to $t_{1 / 2(2)}$ values from 11.5 to 102.3 days for Region 2.

In contrast to Risperidone, polymer degradation in Placebo and Olanzapine microspheres followed first order kinetics (Figure 3, Table 2). The rate of polymer degradation for the Placebo was low, with a $k$ value of $0.003 /$ day. Incorporation of $1 \%$ Olanzapine into the PLGA polymer led to a greater than 10 -fold increase in degradation rate (0.047/day) after which the rate nearly doubled to reach $0.077 /$ day with $5 \%$ loading and remained relatively constant between 5 and 15\% loading. An excellent correlation, $R^{2}>$ 0.95 , was obtained for the first order rate constant, $k$, at all drug loadings of Olanzapine. Similar to the trend noted with the rate constant, a half-life value or approximately 14 days was calculated for $1 \%$ drug load and dropped to around 8 days for loadings between 5 and $15 \%$ of Olanzapine.

3.2.4. Effect of Drug Loading on Polymer Molecular Weight during Manufacture. Unlike Risperidone and Olanzapine, no lowering of polymer molecular weight was observed in

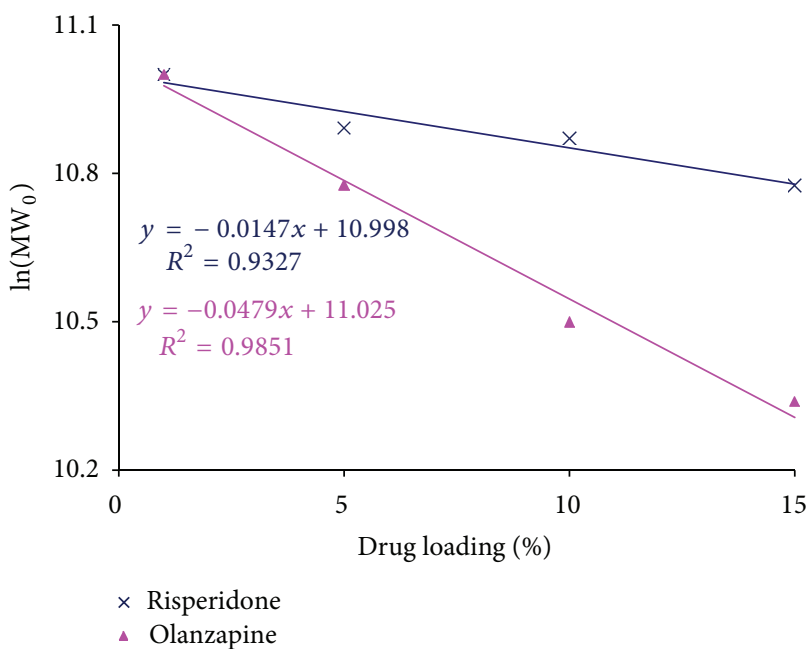

FIGURE 4: Relationship between drug loading and molecular weight reduction in PLGA microspheres after encapsulation of Risperidone and Olanzapine.

the Placebo microsphere during manufacture; there was no difference between $\mathrm{MW}_{\text {initial }}$ and $\mathrm{MW}_{0}$ (Figures 2-3). In contrast, Risperidone and Olanzapine had a noticeable influence on the polymer during the manufacturing process. Olanzapine, a secondary amine, being more basic and nucleophilic when compared to Risperidone, caused a greater lowering in polymer molecular weight during manufacture. From an initial molecular weight of $75 \mathrm{kDa}\left(\mathrm{MW}_{\text {initial }}\right)$, a decrease in polymer molecular weight postmanufacture $\left(\mathrm{MW}_{0}\right)$ was observed with all the drug loaded microspheres (Figures 2-3). This decrease in polymer molecular weight postmanufacture $\left(\mathrm{MW}_{0}\right)$ could be fitted to a first order kinetic model (Figure 4).

A closer inspection of Figure 4 reveals that, at 1\% loading, $\mathrm{MW}_{0}$ was similar for Olanzapine and Risperidone. However, at higher loadings, incorporation of Olanzapine caused a steeper drop in the molecular weight of PLGA; that is, $\mathrm{MW}_{0 \text { (Olanzapine) }}>\mathrm{MW}_{0 \text { (Risperidone) }}$ (Figure 4). Given that Olanzapine is a stronger base and nucleophile, the reaction with an acidic polymer like PLGA was not unexpected. As such it was noted that, over a $1-15 \%$ drug loading range, the value of the slope for Olanzapine was more than three times that observed with Risperidone. The semilogarithmic plot of $\mathrm{MW}_{0}$ values for Risperidone and Olanzapine as a function of loading had a goodness of fit $R^{2}>0.9$ between 1 and $15 \%$ loading for both drugs, indicating that there is a strong 


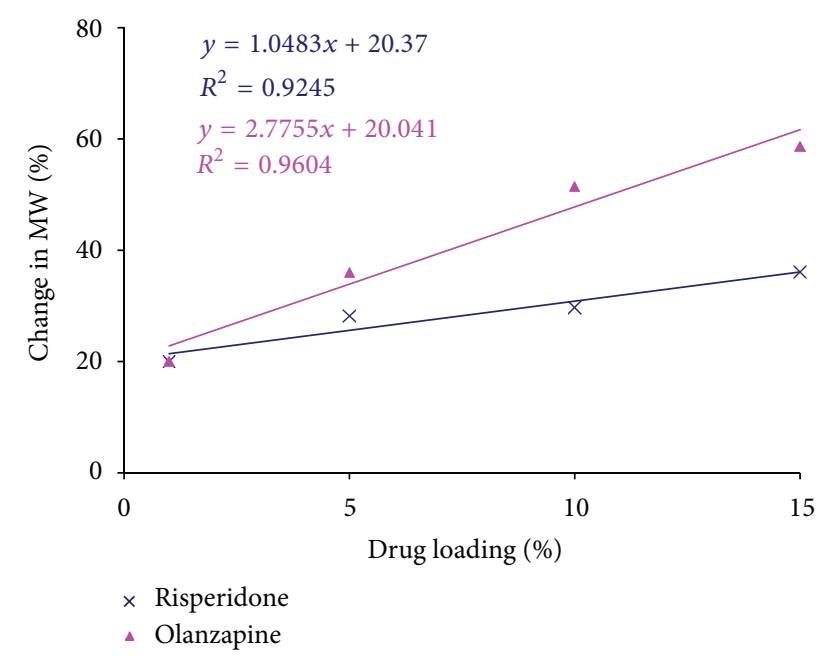

Figure 5: Effect of Risperidone and Olanzapine on \% change in molecular weight during manufacture.

correlation between the amount of drug encapsulated in the microspheres and molecular weight loss during manufacture. Though the intercept values are similar, the slopes for Olanzapine and Risperidone seen in Figure 4 are vastly different. These data affirm that the impact of Olanzapine on the degradation of the $65: 35$ PLGA polymer is at least three times higher than Risperidone and were attributed to the catalytic activity of two tertiary amines and a secondary amine in Olanzapine. In comparison, Risperidone does not contain a secondary amine but has two tertiary amines. Since secondary amines are known to be more reactive than tertiary amines, the presence of this functional group in Olanzapine reacted more strongly with the PLGA polymer.

Interestingly, a linear relationship was also observed when the $\%$ change in molecular weight during manufacture was plotted against \% drug loading (Figure 5, (2)):

$\%$ change in mol. wt. during manufacture

$$
=\frac{\left(\mathrm{MW}_{\text {initial }}-\mathrm{MW}_{0}\right)}{\mathrm{MW}_{\text {initial }}} \times 100 .
$$

Similar to the plot described in Figure 4, the \% change in molecular weight with Olanzapine was nearly three times greater than with Risperidone (Figure 5). As such, the \% change in molecular weight ranged between 20 and 36\% at 1$15 \%$ loadings of Risperidone and $20-59 \%$ at similar loadings of Olanzapine. The large values of \% change in molecular weight observed with Olanzapine suggest that it had a much more debilitating effect on the PLGA polymer. Data analysis of Figure 5 revealed a strong correlation $\left(R^{2}>0.9\right)$ between 1 and 15\% loading for Risperidone as well as Olanzapine, indicating that there was a good relationship between the amount of drug encapsulated in the microspheres and the \% change in molecular weight loss during manufacture.

3.2.5. Extent of Polymer Degradation due to Risperidone and Olanzapine. To gain a better understanding of the extent to which Risperidone and Olanzapine degrade the 65 : 35 PLGA

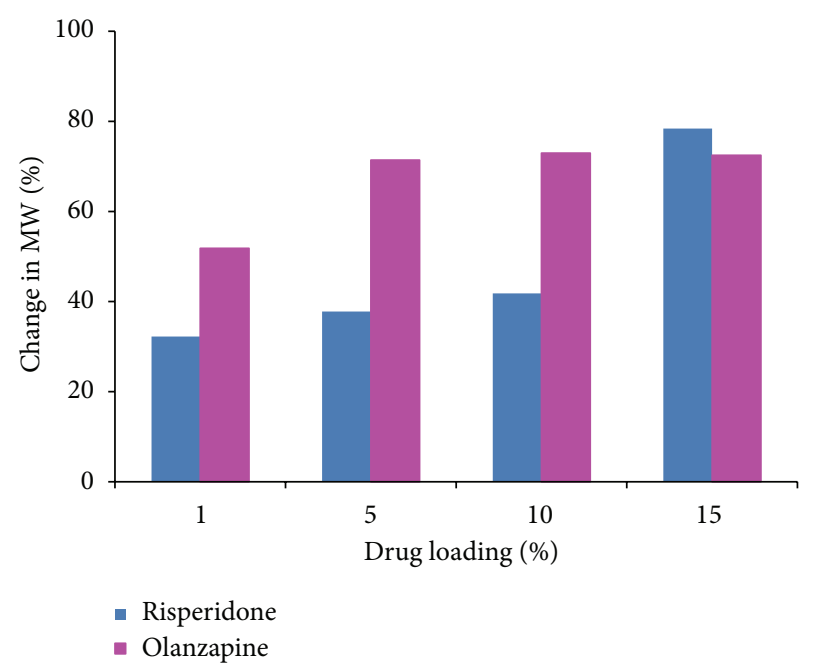

FIGURE 6: Effect of Risperidone and Olanzapine on \% change in molecular weight after 15 days.

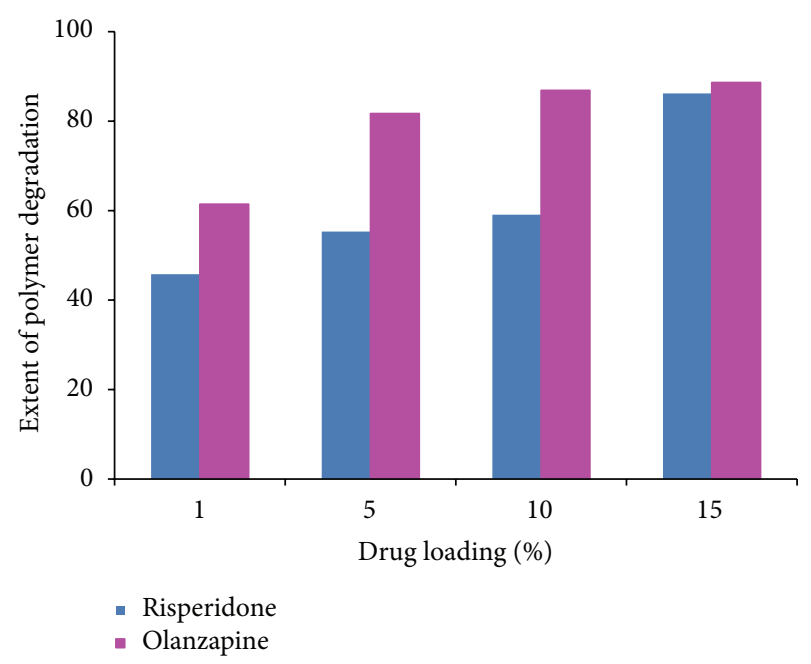

Figure 7: Extent of polymer degradation due to Risperidone and Olanzapine.

polymer when incubated in $0.02 \mathrm{M}$ phosphate buffer saline $\mathrm{pH} 7.2$ containing $0.05 \%$ Tween 80 , at $37^{\circ} \mathrm{C}$, the molecular weight at day $15\left(\mathrm{MW}_{15}\right)$ was compared with the polymer molecular weight of the microspheres $\left(\mathrm{MW}_{0}\right)$ (equation (3), Figure 6), as well as the initial polymer molecular weight $\left(\mathrm{MW}_{\text {initial }}\right)$ (equation (4), Figure 7):

$\%$ change in mol. wt. after 15 days

$$
=\frac{\left(\mathrm{MW}_{0}-\mathrm{MW}_{15}\right)}{\mathrm{MW}_{0}} \times 100 \text {. }
$$

Since the molecular weight of the polymer in the microspheres $\left(\mathrm{MW}_{0}\right)$ varied based on the drug loading (Figures $2-3)$, the $\%$ change in molecular weight that occurred as a result of in vitro degradation experiments through day 15 was calculated as described in (3) and plotted (Figure 6). At first glance, the high values of $\%$ change in molecular 
weight with Olanzapine are clearly obvious. In fact, even at the low loading of $1 \%$ Olanzapine, nearly $50 \%$ of the polymer was hydrolyzed through day 15 of the in vitro study. For $5-15 \%$ loadings, the \% change in molecular weight after 15 days of degradation had reached a plateau between 71 and $73 \%$ and was attributed to the similarity in rate constants at higher loadings of Olanzapine (Table 2). On the other hand, a slower drop in molecular weight was noted with Risperidone microspheres. At the low and moderate loadings of 1, 5, and $10 \%$, the $\%$ change in molecular weight ranged between 32 and $41 \%$. However, at the highest loading (15\%), the \% change increased significantly to $78 \%$, slightly more than that noted with the corresponding strength of Olanzapine.

Another parameter, that is, the extent of polymer degradation, was calculated to provide an insight to the extent which Risperidone and Olanzapine degrade the polymer. In essence, it is a comparison of the raw polymer molecular weight $\left(\mathrm{MW}_{\text {initial }}\right)$ against the molecular weight after the 15 day degradation study $\left(\mathrm{MW}_{15}\right)$ and calculated as described in (4) below

Extent of polymer degradation

$$
=\frac{\left(\mathrm{MW}_{\text {initial }}-\mathrm{MW}_{15}\right)}{\mathrm{MW}_{\text {initial }}} \times 100 \text {. }
$$

From Figure 7, a 61\% drop in molecular weight at day 15 was observed with $1 \%$ Olanzapine loading, and values reached a plateau at drug loads of $10-15 \%$; that is, extent of polymer degradation was around $86-88 \%$. For Risperidone microspheres, the drop appeared more gradual, increasing from $45 \%$ (1\% drug load) to $60 \%$ (10\% drug load) after which a sudden spike in degradation was noted at $15 \%$ Risperidone $(\sim 86 \%)$. A comparison of the data in Figure 7 revealed that, at similar loadings of Risperidone and Olanzapine, the extent of polymer degradation was nearly $15-30 \%$ greater with Olanzapine. For instance, at $1 \%$ loading of Risperidone, the molecular weight at day $15\left(\mathrm{MW}_{15}\right)$ had dropped to $45 \%$ of its initial value $\left(\mathrm{MW}_{\text {initial }}\right)$, whereas the drop with $1 \%$ Olanzapine was much steeper $(\sim 61 \%)$, that is, a difference of around $15 \%$. At loadings between 5 and $10 \%$, the difference was more marked and ranged around $25-30 \%$; a greater drop in $\mathrm{MW}_{15}$ was observed with Olanzapine. Interestingly, the extent of degradation at $10 \%$ loading of Risperidone was quite similar to that observed with $1 \%$ loading of Olanzapine. However, the $\mathrm{MW}_{15}$ values for Risperidone and Olanzapine were almost equal (Figures 6-7). Upon closer scrutiny of the data at day 15, it was clear that $M_{15}$ values at 10 and $15 \%$ Olanzapine had reached a plateau due to extensive breakdown of the polymer.

In general, the findings presented in Sections 3.2.1 to 3.2.5 of this study are the result of a concerted effort to enable a systematic and efficient formulation development of these molecules in a biodegradable polymer and, consequently, select an appropriate sustained release injectable microsphere dosage form that could provide drug release for 2 or more weeks.
The rationale for implementing such a systematic formulation development approach is twofold and offers several advantages.

(i) Ensuring That the Right Amount of Drug Loading Is Present in the Delivery Matrix throughout the Intended Duration of Action. The amount of drug loading in a delivery matrix is known to be one of the key modulators of the rate and duration of drug release [29]. For example, dosage forms that contain high drug loading release drug faster due to low polymer content in the dosage form (Figures 2-3). Conversely, a higher polymer to drug ratio should have the opposite effect. For drugs like Risperidone and Olanzapine, using a systematic formulation development strategy would enhance formulation development efforts by: (a) ensuring that sufficient levels of the atypical antipsychotic are present in the delivery matrix and available throughout the duration of action in vivo $[27,28]$ and (b) eliminating the need for daily oral dosing and improving patient compliance [6]. Hence, biodegradable microspheres of Risperidone and Olanzapine were evaluated at a range of drug loadings.

(ii) Determining the Suitability of the Polymer as a Delivery Matrix. Both Risperidone and Olanzapine are weakly basic nucleophiles that will be encapsulated in a polyester based polymer containing carboxylic end groups [COOH]. Due to the known reactivity of other basic drugs with the PLGA polymer $[12,19]$, it is critical to understand the impact of Risperidone and Olanzapine on degradation of the 65:35 PLGA polymer, as it influences the dosage form performance in vivo. Ensuring that polymer properties like molecular weight are suitable for incorporating a basic drug while retaining sustained delivery characteristics are some important aspects that should be assessed in parallel with dosage form design. This allows for the selection of a suitable polymer for encapsulating moieties like basic drugs that are known to react with poly(ester) polymers like PLA or PLGA. Hence, assessing the impact of varying drug loadings on polymer behavior is a rational approach in the design of polymeric formulations for sustained delivery of atypical antipsychotics and was implemented in this study.

As per the formulation rationale presented above, drug loadings between 1 and 15\% provided a critical insight about the drug: polymer ratio required for dosage form development. Additionally, since the commercially available Risperidone microspheres contain 38\% drug load, a 35\% loading of drug for Risperidone was also assessed [30]. Based on these molecular weight results in this study, it would be unwise to neglect the impact of a basic drug on the PLGA polymer during the manufacturing process, thereby reinforcing the importance of understanding chemical properties of drugs and their impact on the polymer.

3.3. Effect of $p H$ and Temperature on Polymer Degradation. To obtain a better understanding of the mechanism in which these atypical antipsychotics catalyze polymer hydrolysis, $\mathrm{pH}$ and temperature dependency of the degradation process was assessed. In comparing the effect of Risperidone and Olanzapine loadings on polymer degradation, the former 


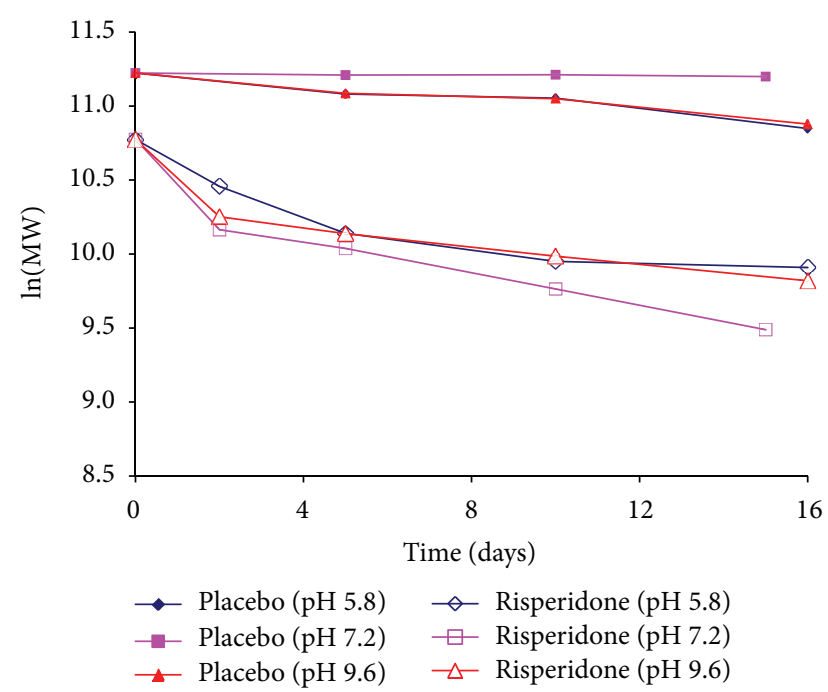

FIGURE 8: Effect of $\mathrm{pH}$ on degradation of Risperidone microspheres at $37^{\circ} \mathrm{C}$.

has a lower effect on degradation. Hence, for the remainder of the experimental study, Risperidone was chosen as the preferred candidate to evaluate the mechanism by which basic nucleophiles cause degradation of the PLGA polymer and was selected as the model drug for additional studies.

3.3.1. Effect of $p H$ on Degradation of Risperidone PLGA Microspheres. To gain insight into the manner in which Risperidone influences polymer degradation, degradation studies were performed at acidic, neutral, and alkaline $\mathrm{pH}(\mathrm{pH} 5.8$, 7.2, and 9.6, resp., at $37^{\circ} \mathrm{C}$ in $0.02 \mathrm{M}$ PBS containing $0.05 \%$ Tween 80 ) and compared against a control (Placebo microspheres).

Figure 8 illustrates the effect of $\mathrm{pH}$ on the degradation of Placebo and Risperidone microspheres. Since the degradation rate reached a plateau between 15 and 35\% loading (Table 2), the higher loading of Risperidone (35\%) was selected to ensure greater sensitivity at the $3 \mathrm{pH}$ values used in the degradation study. From the semilogarithmic plot, it is evident that the Placebo microspheres degrade slightly faster under acidic and alkaline $\mathrm{pH}$ values but continue to follow pseudo first order kinetics. Interestingly, the profiles at $\mathrm{pH}$ 5.8 and 9.6 are almost superimposable, with the extent of degradation (3) around $30 \%$ at both conditions, but $2 \%$ at neutral $\mathrm{pH}$. As such, the degradation profiles at the acidic and alkaline conditions can be explained using the general acid-base catalysis where ester hydrolysis is enhanced by the presence of $\mathrm{H}+$ or $\mathrm{OH}-$ ions in the aqueous media. Thus, the mechanism of PLGA hydrolysis in acidic or alkaline media is different from that observed in neutral media [31].

When compared to the Placebo microspheres, the presence of Risperidone impacted the reaction profile and increased the rate of degradation of the polymer at all the $\mathrm{pH}$ values studied. A biphasic degradation profile was observed with the Risperidone microspheres, similar to that presented in Figure 2, at the neutral and alkaline conditions in that a rapid initial decrease in polymer molecular weight was

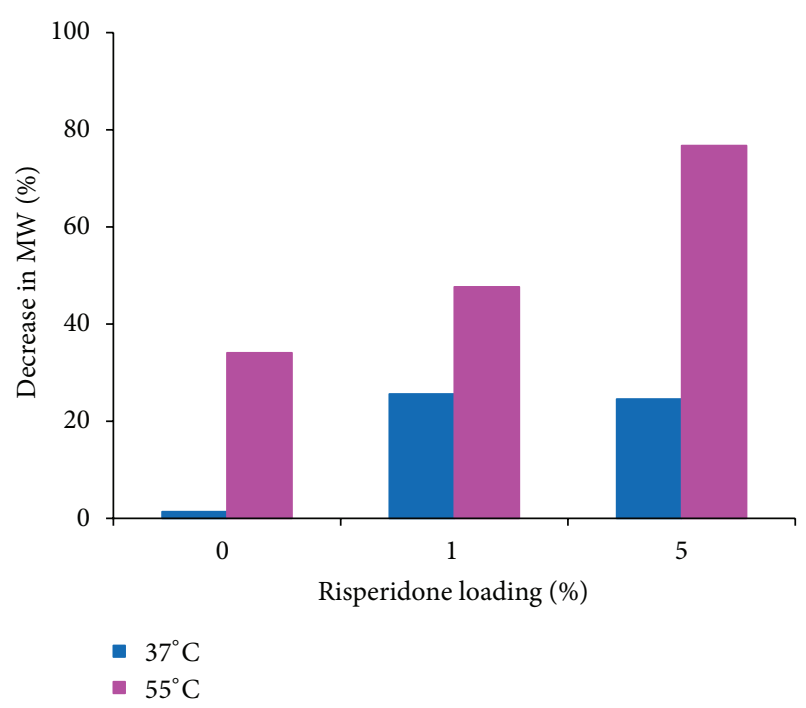

FIGURE 9: Effect of temperature on the \% decrease in molecular weight of Risperidone microspheres after 2 days of incubation at 37 and $55^{\circ} \mathrm{C}$.

observed at day 2 . In contrast, the sharp dip in molecular weight at day 2 was not noted at the acidic $\mathrm{pH}$ and was attributed to the neutralization of the weakly basic drug. After day 2, the profiles at the acidic and alkaline $\mathrm{pH}$ values were almost superimposable, similar to that observed with the Placebo microspheres. However, the reaction rate slowed down slightly when compared to that at neutral $\mathrm{pH}$, with the extent of degradation (3) around $75 \%$ at the acidic and alkaline $\mathrm{pH}$ values $(\sim 82 \%$ at neutral $\mathrm{pH})$. The results at $\mathrm{pH}$ values 5.8 and 9.6 implied that degradation of the polymer occurred at a similar rate with the free base and the salt form of Risperidone, indicating that the reaction did not proceed via general acid-base catalysis, but a combination of specific base and/or nucleophilic catalysis.

\subsubsection{Effect of Temperature on Degradation of Risperidone} Microspheres. The effect of temperature on polymer degradation of Risperidone microspheres was investigated at 0 (Placebo), 1 , and $5 \%$ loadings at $37^{\circ} \mathrm{C}$ and $55^{\circ} \mathrm{C}$ in $0.02 \mathrm{M}$ $\mathrm{PBS} \mathrm{pH} 7.2$, and a comparison of the \% decrease in polymer molecular weight after 2 days is shown (Figure 8). For the Placebo microspheres ( $0 \%$ loading), the rate of polymer degradation increased and followed pseudo first order kinetics. Indeed, the $\%$ decrease in molecular weight was well over $30 \%$ after just 2 days at $55^{\circ} \mathrm{C}$, compared to meager $1 \%$ at $37^{\circ} \mathrm{C}$. Likewise, the degradation profiles for Risperidone microspheres at $55^{\circ} \mathrm{C}$ were biphasic, similar to what was observed at $37^{\circ} \mathrm{C}$, albeit at a much faster rate (data not shown). After two days of incubation, the extent of reduction in the molecular weight was much higher at $55^{\circ} \mathrm{C}, 46 \%$ for the $1 \%$ loading, and $76 \%$ for the $5 \%$ loading. In comparison, the 2 day molecular weight reduction at $37^{\circ} \mathrm{C}$ was nearly $25 \%$ for 1 and 5\% Risperidone loadings, indicating that temperature has a pronounced effect on polymer degradation, especially at increased loadings. Overall, polymer degradation was accelerated at the higher temperature with a greater extent 
in molecular weight reduction observed with increased drug loading (Figure 9).

It has been well established that the rate of a chemical reaction is influenced by temperature. With biodegradable polymers like PLGA or PLA, the relationship between temperature and polymer degradation has been explored by a few authors. For example, Buchholz examined the temperature dependency of neat polymer degradation at 37 and $80^{\circ} \mathrm{C}$ [32]. Another report commented on the polymer degradation profile of neat PLA at a temperature of $90^{\circ} \mathrm{C}$ [33]. From these studies, it was clear that PLGA and PLA degradation rate was enhanced at higher temperatures and is beneficial to researchers in this area as it allows a substantial reduction in the time and labor needed to assess polymer degradation [34]. Additionally, these studies indicated that increasing the temperature of degradation experiment did not alter the mechanism of polymer degradation. The elevated temperature experiments (accelerated tests) were later extrapolated for their use as a rapid evaluation technique for drug loaded polymeric dosage forms. The success of the devised technique, as noted by the interest in accelerated testing of biodegradable microspheres, is evidenced by a growing number of publications utilizing this methodology $[31,35,36]$. Additionally, accelerated tests have proven to be a useful tool to predict real-time in vitro behavior of biodegradable microspheres.

In the current study, since temperature effects on polymer degradation were expected to be profound, lower loadings of Risperidone microspheres (1 and 5\%) were selected for evaluation and the results were compared with a control (Placebo microspheres). The results obtained from Figure 9 suggest that the elevated temperatures can be used to accelerate polymer degradation and can be used to evaluate the effect of drug loaded microspheres encapsulated with a basic drug like Risperidone in a rapid manner. These findings are similar to previous reports on neat PLA and PLGA polymers [32-34]. Additionally, no change in the biphasic degradation profile was observed with Risperidone microspheres, further corroborating previous findings from earlier experiments (Figure 2).

\section{Conclusions}

While the biodegradability, biocompatibility, and nonimmunogenicity of PLGA polymers have rendered them suitable for a variety of surgical and medical applications, including dosage forms, the degradative effects of basic and nucleophilic drugs Risperidone and Olanzapine on the polymer diminish their utility with respect to providing sustained levels of encapsulated therapeutic agents over extended intervals. Incorporation of such candidates into PLGA, even at low drug loadings, will greatly enhance polymer degradation and thereby influence formulation performance, in vitro or in vivo. Further, the effect of buffer $\mathrm{pH}$ and temperature on polymer molecular weight and degradation should also be considered. All these factors need to be considered while developing PLGA formulations containing basic and nucleophilic drugs for sustained drug delivery.

\section{Conflict of Interests}

The authors declare that there is no conflict of interests regarding the publication of this paper.

\section{Acknowledgments}

The research described in this paper was performed while the authors were affiliated with the University of Kentucky, Lexington, KY. The authors wish to thank Oakwood Labs, Oakwood, $\mathrm{OH}$, and the Graduate School, University of Kentucky, Lexington, KY, for their financial support.

\section{References}

[1] T. H. Kim, H. H. Jiang, C. W. Park et al., "PEGylated TNFrelated apoptosis-inducing ligand (TRAIL)-loaded sustained release PLGA microspheres for enhanced stability and antitumor activity," Journal of Controlled Release, vol. 150, no. 1, pp. 63-69, 2011.

[2] J. He, M. Feng, X. Zhou et al., "Stabilization and encapsulation of recombinant human erythropoietin into PLGA microspheres using human serum albumin as a stabilizer," International Journal of Pharmaceutics, vol. 416, no. 1, pp. 69-76, 2011.

[3] N. Umeki, T. Sato, M. Harada et al., "Preparation and evaluation of biodegradable microspheres containing a new potent osteogenic compound and new synthetic polymers for sustained release," International Journal of Pharmaceutics, vol. 392, no. 1-2, pp. 42-50, 2010.

[4] D. Bodmer, T. Kissel, and E. Traechslin, "Factors influencing the release of peptides and proteins from biodegradable parenteral depot systems," Journal of Controlled Release, vol. 21, no. 1-3, pp. 129-137, 1992.

[5] P. P. DeLuca, R. C. Mehta, A. G. Hausberger, and B. C. Thanoo, "Biodegradable polyesters for drug and polypeptide delivery," in Polymer Delivery Systems, Properties and Applications, ACS Symposium Series, American Chemical Society, Washington, DC, USA, 1993.

[6] S. D'Souza, J. Faraj, and P. Deluca, "Microsphere delivery of Risperidone as an alternative to combination therapy," European Journal of Pharmaceutics and Biopharmaceutics, vol. 85, no. 3, pp. 631-639, 2013.

[7] H. Okada, Y. Doken, Y. Ogawa, and H. Toguchi, "Preparation of three-month depot injectable microspheres of leuprorelin acetate using biodegradable polymers," Pharmaceutical Research, vol. 11, no. 8, pp. 1143-1147, 1994.

[8] G. Ploussard and P. Mongiat-Artus, "Triptorelin in the management of prostate cancer," Future Oncology, vol. 9, no. 1, pp. 93102, 2013.

[9] D. Fontana, M. Mari, A. Martinelli et al., "3-Month formulation of goserelin acetate ('Zoladex' 10.8-mg depot) in advanced prostate cancer: Results from an Italian, open, multicenter trial," Urologia Internationalis, vol. 70, no. 4, pp. 316-320, 2003.

[10] Y. Sun, J. Wang, X. Zhang et al., "Synchronic release of two hormonal contraceptives for about one month from the PLGA microspheres: in vitro and in vivo studies," Journal of Controlled Release, vol. 129, no. 3, pp. 192-199, 2008.

[11] B. H. Woo, J. W. Kostanski, S. Gebrekidan, B. A. Dani, B. C. Thanoo, and P. P. DeLuca, "Preparation, characterization and in vivo evaluation of 120-day poly(D,L-lactide) leuprolide microspheres," Journal of Controlled Release, vol. 75, no. 3, pp. 307-315, 2001. 
[12] H. V. Maulding, T. R. Tice, D. R. Cowsar, J. W. Fong, J. E. Pearson, and J. P. Nazareno, "Biodegradable microcapsules: acceleration of polymeric excipient hydrolytic rate by incorporation of a basic medicament," Journal of Controlled Release, vol. 3, no. 1-4, pp. 103-117, 1986.

[13] Y. Cha and C. G. Pitt, "The acceleration of degradation-controlled drug delivery from polyester microspheres," Journal of Controlled Release, vol. 8, no. 3, pp. 259-265, 1989.

[14] A. Kishida, S. Yoshioka, S. Izumikawa, Y. Aso, and Y. Takeda, "Formulation-assisted biodegradable polymer matrices," Chemical and Pharmaceutical Bulletin, vol. 37, pp. 1954-1956, 1989.

[15] R. Bodmeier and H. G. Chen, "Evaluation of biodegradable poly(lactide) pellets prepared by direct compression," Journal of pharmaceutical sciences, vol. 78, no. 10, pp. 819-822, 1989.

[16] S. D’Souza, J. A. Faraj, S. Giovagnoli, and P. P. Deluca, "IVIVC from long acting olanzapine microspheres," International Journal of Biomaterials, vol. 2014, Article ID 407065, 11 pages, 2014.

[17] S. Takada, T. Kurokawa, K. Miyazaki, S. Iwasa, and Y. Ogawa, "Sustained release of a water-soluble GP IIb/IIIa antagonist from copoly(d,l-lactic/glycolic)acid microspheres," International Journal of Pharmaceutics, vol. 146, no. 2, pp. 147-157, 1997.

[18] S. J. Siegel, J. B. Kahn, K. Metzger, K. I. Winey, K. Werner, and N. Dan, "Effect of drug type on the degradation rate of PLGA matrices," European Journal of Pharmaceutics and Biopharmaceutics, vol. 64, no. 3, pp. 287-293, 2006.

[19] M. Miyajima, A. Koshika, J. Okada, and M. Ikeda, "Effect of polymer/basic drug interactions on the two-stage diffusioncontrolled release from a poly(L-lactic acid) matrix," Journal of Controlled Release, vol. 61, no. 3, pp. 295-304, 1999.

[20] N. Wakiyama, K. Juni, and M. Nakano, "Preparation and evaluation in vitro of polylactic acid microspheres containing local anesthetics," Chemical and Pharmaceutical Bulletin, vol. 29, no. 11, pp. 3363-3368, 1981.

[21] N. Wakiyama, K. Juni, and M. Nakano, "Influence of physicochemical properties of polylactic acid on the characteristics and in vitro release patterns of polylactic acid microspheres containing local anesthetics," Chemical and Pharmaceutical Bulletin, vol. 30, no. 7, pp. 2621-2628, 1982.

[22] N. Wakiyama, K. Juni, and M. Nakano, "Preparation and evaluation in vitro and in vivo of polylactic acid microspheres containing dibucaine," Chemical \& Pharmaceutical Bulletin, vol. 30, no. 10, pp. 3719-3727, 1982.

[23] T. S. Harrison and K. L. Goa, "Long-acting Risperidone: a review of its use in Schizophrenia," CNS Drugs, vol. 18, no. 2, pp. 113$132,2004$.

[24] H. He and J. S. Richardson, "A pharmacological, pharmacokinetic and clinical overview of risperidone, a new antipsychotic that blocks serotonin 5-HT2 and dopamine D2 receptors," International Clinical Psychopharmacology, vol. 10, no. 1, pp. 1930, 1995.

[25] N. Bhana and C. M. Perry, "Olanzapine: a review of its use in the treatment of bipolar I disorder," CNS Drugs, vol. 15, no. 11, pp. 871-904, 2001.

[26] P. L. McCormack and L. R. Wiseman, "Olanzapine: a review of its use in the management of bipolar I disorder," Drugs, vol. 64, no. 23, pp. 2709-2726, 2004.

[27] S. D’Souza, J. A. Faraj, S. Giovagnoli, and P. P. DeLuca, "Preparation, characterization and in vivo evaluation of Olanzapine Poly(D,L-lactide-co-glycolide) (PLGA) microspheres," Journal of Pharmaceutics, vol. 2013, Article ID 831381, 9 pages, 2013.
[28] S. D’Souza, J. A. Faraj, S. Giovagnoli, and P. P. DeLuca, "Development of risperidone PLGA microspheres," Journal of Drug Delivery, vol. 2014, Article ID 620464, 11 pages, 2014.

[29] A. O. Eniola and D. A. Hammer, "Characterization of biodegradable drug delivery vehicles with the adhesive properties of leukocytes II: effect of degradation on targeting activity," Biomaterials, vol. 26, no. 6, pp. 661-670, 2005.

[30] M. Ramstack, G. Grandolfi, and E. Mannaert, "Long-acting Risperidone: prolonged release injectable delivery of Risperidone using Medisorb microsphere technology," Biological Psychiatry, vol. 53, abstract 547, 2003.

[31] S. S. D’Souza, J. A. Faraj, and P. P. DeLuca, "A model-dependent approach to correlate accelerated with real-time release from biodegradable microspheres," AAPS PharmSciTech, vol. 6, no. 4, article no. 70, 2005.

[32] B. Buchholz, "Accelerated degradation test on resorbable polymers," in Degradation Phenomena of Polymeric Biomaterials, p. 67, Springer, New York, NY, USA, 1992.

[33] J. E. Bergsma, F. R. Rozema, R. R. M. Bos, G. Boering, C. A. P. Joziasse, and A. J. Pennings, "In vitro predegradation at elevated temperatures of poly(lactide)," Journal of Materials Science: Materials in Medicine, vol. 6, no. 11, pp. 642-646, 1995.

[34] C. M. Agrawal, D. Huang, J. P. Schmitz, and K. A. Athanasiou, "Elevated temperature degradation of a 50:50 copolymer of PLA-PGA," Tissue Engineering, vol. 3, no. 4, pp. 345-352, 1997.

[35] M. Shameem, H. Lee, and P. P. DeLuca, "A short-term (accelerated release) approach to evaluate peptide release from PLGA depot formulations," AAPS PharmSci, vol. 1, no. 3, article 7, 1999.

[36] S. D’Souza, J. A. Faraj, R. Dorati, and P. P. DeLuca, "A short term quality control tool for biodegradable microspheres," AAPS PharmSciTech, vol. 15, no. 3, pp. 530-541, 2014. 

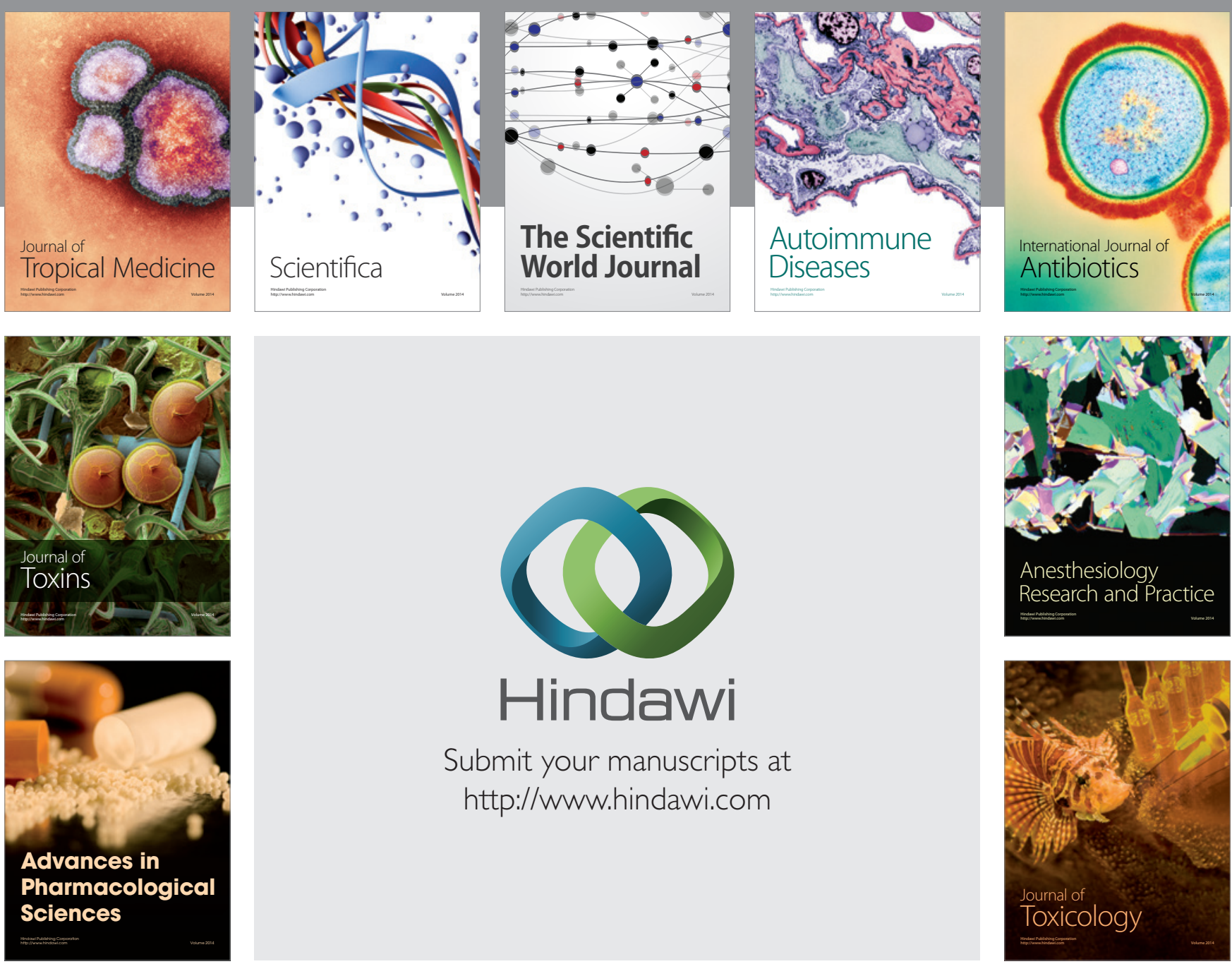

\section{Hindawi}

Submit your manuscripts at

http://www.hindawi.com
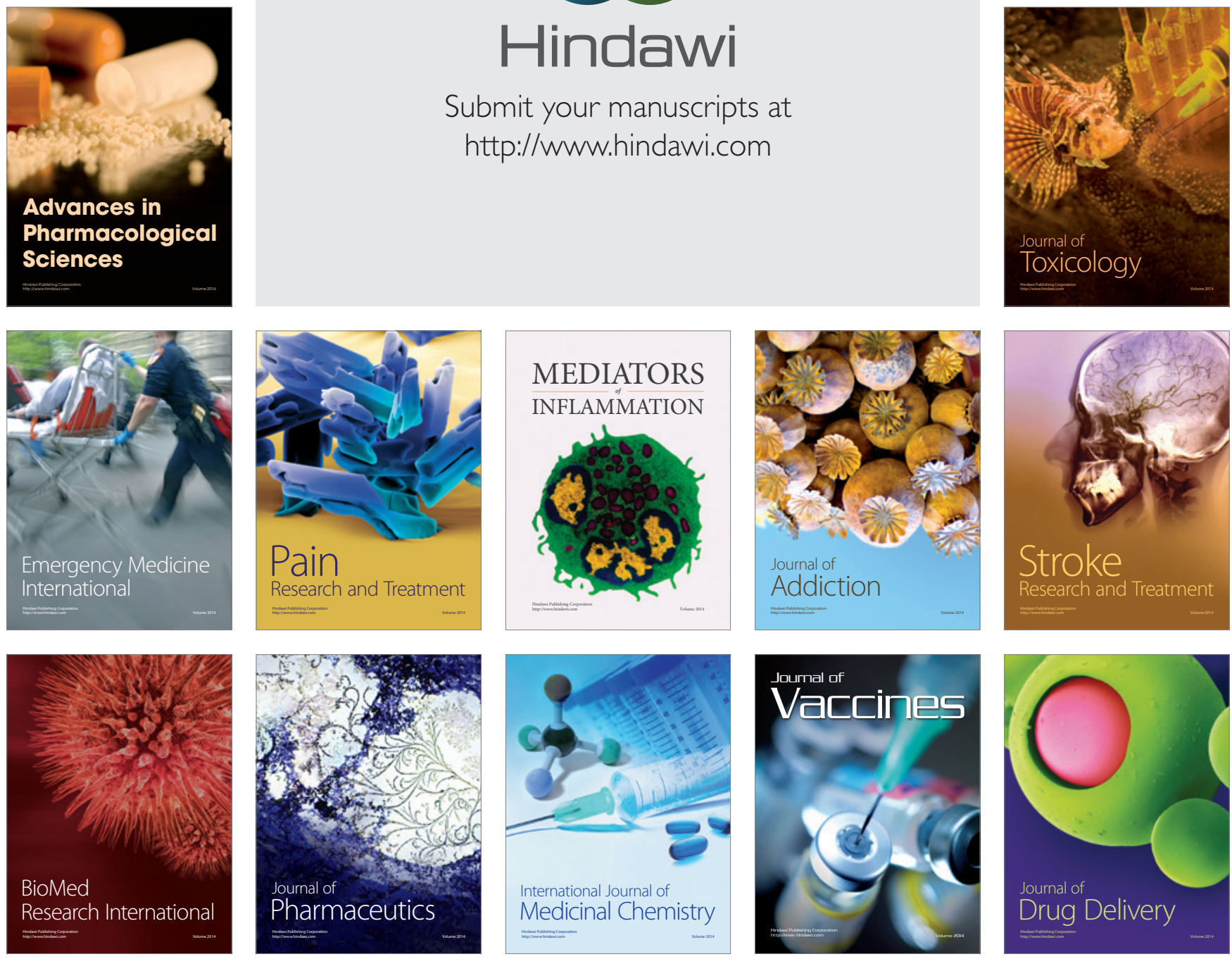